Department of Family Medicine, Faculty of Health Sciences, University of Pretoria

T M Rossouw, MB ChB, MPhil, MPH, PhD

Private practice, Pretoria

M E Botes, MB ChB

Department of Clinical Medicine, Faculty of Health Sciences, University of the Witwatersrand, Johannesburg

F Conradie, MB BCh, Dip HIV Man, Dip Tropical Medicine and Hygiene

Corresponding author: T M Rossouw (theresa.rossouw@up.ac.za)

Lipodystrophy is a well-recognised adverse effect of HIV and antiretroviral therapy, with certain antiretrovirals, specifically thymidine analogues, implicated in the aetiology and pathogenesis. Lipodystrophy is often accompanied by metabolic complications, such as hyperlipidaemia and insulin resistance, which increase risk for cardiovascular disease. There are limited data on the effect of treatment modification, pharmacological interventions and surgical management on this condition.

Here we summarise the latest data on lipodystrophy, with the aim of facilitating informed decision-making in managing this condition. In light of the absence of cost-effective measures to treat lipoatrophy and lipohypertrophy, prevention remains the best option; we recommend targeted annual screening. Healthcare workers should be sensitised to early detection in patients on thymidine-based regimens, and affected patients should be switched to an appropriate regimen as soon as feasible. There is no evidence to support the use of new-generation ARVs, except in patients with significant hypercholesterolaemia, where atazanavir and raltegravir may present better options.

S Afr J HIV Med 2013;14(1):29-33. DOI:10.7196/SAJHIVMED.871

The lipodystrophy syndrome is a well-recognised phenomenon in HIV-1-infected patients receiving antiretroviral therapy (ART). The syndrome is characterised by body habitus changes, most commonly a combination of lipoatrophy (LA) (loss of peripheral subcutaneous adipose tissue (SAT), usually in the face, limbs and buttocks) and lipohypertrophy (LH) (visceral adipose tissue (VAT) accumulation, gynaecomastia and, in some cases, lipomatosis, especially in the dorsocervical area, known as a 'buffalo hump').

Even though the aetiology remains unclear, the following factors havebeen implicated in the developmentoflipodystrophy: HIV itself, older age, female sex, genetic parameters and ART. ${ }^{[1]}$ There seems to be consensus that peripheral LA and central LH have the same causes (HIV and ART), but are likely to be related to different fat depot physiologies. ${ }^{[2]}$ It is speculated that LA is linked to severe mitochondrial dysfunction, oxidative stress and inflammation, while hypertrophy is related to mild mitochondrial dysfunction and cortisol activation, promoted by inflammation. Importantly, both LA in the lower part of the body and abdominal LH have been associated with metabolic changes akin to the metabolic syndrome, particularly dyslipidaemia and insulin resistance. ${ }^{[3]}$

Recent reports in the popular press have highlighted the psychological and social distress that patients experience as a result of lipodystrophy. Often, patients are left on offending regimens for too long, causing extreme and often irreversible body changes. It is imperative that healthcare workers familiarise themselves with this entity and understand the treatment options available to patients. Here we summarise the latest data on the topic, with the aim of facilitating informed decision-making in managing this condition.

\section{Role of ART}

Data on the effect of ART on lipodystrophy exist for the older nucleoside reverse transcriptase inhibitors (NRTIs) and unboosted protease inhibitors (PIs), but seem to be conflicting for the non-nucleoside reverse transcriptase inhibitors (NNRTIs) and newer PIs, and are mostly unavailable for the new drug classes. Many studies have implicated stavudine (d4T) and zidovudine (AZT) in the development of lipodystrophy and this is generally uncontested. Abacavir (ABC) and tenofovir (TDF) have been shown to have minimal effect. ${ }^{[6]}$ Initial studies did not reveal a role for efavirenz (EFV) in lipodystrophy, but the recent ACTG A5142 study ${ }^{[6]}$ found that LA was indeed more relevant in EFVtreated patients when compared with boosted lopinavir (LPV/r)treated patients. Another study, however, found only a minimal difference between EFV and another PI, atazanavir (ATV). ${ }^{[]]}$Taken together, these studies suggest that in terms of the development of LA, LPV may have a slight advantage over EFV when combined with TDF (up to 96 weeks) in treatment-naive patients. In terms of lipodystrophy, ATV may have a marginal advantage over EFV when combined with AZT (up to 48 weeks) in treatment-naive 
patients. Importantly, ATV is known to have a better lipid profile than EFV and LPV/r and an argument could therefore be made for its preferential use in patients at high risk for cardiovascular disease.

The 2011 United States Department of Health and Human Services ART guidelines state that an increase in trunk fat has been observed with EFV-, PI-, and raltegravir (RAL)-containing regimens, but that a causal relationship has not been established. ${ }^{[4]}$ There are very few data on the entry inhibitors and integrase inhibitors, although some early data indicate the possibility of an improved metabolic and lipid profile. There are currently no systematic reviews or meta-analyses comparing the effects of different antiretrovirals (ARVs) on lipodystrophy. The comparison of studies is complicated by different measurement standards for lipodystrophy, the use of different race and ethnic groups, and a general lack of control groups.

Table 1 summarises the current knowledge about the effect of different ARVs on lipodystrophy, lipids and glucose metabolism.

It is important to note that the number of patients changing ART regimens because of lipodystrophy appears to be decreasing over time. The Swiss HIV Cohort Study ${ }^{[5]}$ followed 5777 participants who started ART between 2000 and 2006, and compared rates of lipodystrophy between 2000 and 2002, and 2003 and 2006. The findings revealed that $4 \%$ of patients had changed ART regimens due to lipodystrophy during $2000-2002$, whereas only $1 \%$ of patients changed during $2003-2006$. This reduction was attributed to the decreased use of AZT ( $88 \%$ v. $64 \%)$ and d4T $(4.2 \%$ v. $0.7 \%)$ and the increased use of TDF ( $0 \%$ v. $30 \%)$ over this time. ${ }^{[5]}$

The differential metabolic effects of the NNRTIs, NRTIs and PIs are depicted in Fig. 1. There is currently not enough long-term information on entry inhibitors and integrase inhibitors to allow for categorisation.

\section{Metabolic consequences of lipodystrophy}

Lipodystrophy is well known to cause significant psychological distress and has been identified as a risk factor for ART non-adherence. ${ }^{[9,10]}$ There are, however, other consequences that may warrant treatment modification, especially metabolic complications. Fig. 2 shows that increased central fat and decreased limb fat are implicated in the development of metabolic complications. The basic pathology is postulated to be an increased release of cytokines and free fatty acids (FFAs) which, together with decreased adiponectin production by adipose tissue, lead to insulin resistance and triglyceride (TG) deposition in tissues such as the liver, skeletal muscle and heart. It seems that active lipolysis in SAT, combined with impaired fat storage capacity in the subcutaneous depot, drives the deposition of lipids in ectopic sites such as the viscera and other non-adipose sites. This leads to hepatic steatosis and increased lipid content in skeletal muscle, which contribute to systemic metabolic alterations, especially insulin resistance. The high levels of FFAs may also affect pancreatic function and thus contribute to impaired insulin release and a pre-diabetic state.

These metabolic changes ultimately result in impaired glucose tolerance and dyslipidaemia with decreased high-density lipoprotein cholesterol (HDL-C) and increased TGs. Metabolic complications are therefore responsible for increased cardiovascular and hepatic disease risks. These changes have also been linked to premature ageing. The hypothesis has been put forward that chronic HIV infection, combined with the use of some ARVs and lipodystrophy, may accelerate the normal ageing processes and lead to the early development of agerelated co-morbidities. ${ }^{[2]}$

Table 1. The effects of different ARV drugs on fat and metabolism*

\begin{tabular}{|c|c|c|c|c|}
\hline Drug & LA & LH & Dyslipidaemia & Insulin resistance \\
\hline Stavudine $(\mathrm{d} 4 \mathrm{~T})$ & +++ & ++ & ++ & ++ \\
\hline Zidovudine (AZT) & ++ & + & + & ++ \\
\hline Didanosine (DDI) & $+/-$ & $+/-$ & + & + \\
\hline Lamivudine (3TC) & 0 & 0 & + & 0 \\
\hline Abacavir (ABC) & 0 & 0 & + & 0 \\
\hline Tenofovir (TDF) & 0 & 0 & 0 & 0 \\
\hline Emtricitabine (FTC) & 0 & 0 & 0 & 0 \\
\hline Efavirenz (EFV) & $+/-$ & $+/-$ & $++\uparrow \mathrm{HDL}$ & + \\
\hline Nevirapine (NVP) & 0 & 0 & $+\uparrow \mathrm{HDL}$ & 0 \\
\hline Ritonavir (RTV) & $+/-$ & + & +++ & ++ \\
\hline Indinavir (IDV) & $+/-$ & + & + & +++ \\
\hline Lopinavir (LPV) & $+/-$ & + & ++ & ++ \\
\hline Saquinavir (SQV) & $+/-$ & + & $+1-$ & $+/-$ \\
\hline Atazanavir (ATV) & 0 & ++ & $+/-$ & 0 \\
\hline Darunavir (DRV) & 0 & + & $+/-$ & $+/-$ \\
\hline Enfuvirtide (INN) & Insufficient data & Insufficient data & 0 & 0 \\
\hline Maraviroc (MVC) & Insufficient data & Insufficient data & 0 & 0 \\
\hline Raltegravir (RAL) & Insufficient data & Insufficient data & 0 & 0 \\
\hline
\end{tabular}




\begin{tabular}{|c|c|c|c|}
\hline \multirow[t]{8}{*}{$\begin{array}{l}\text { Metabolic } \\
\text { effect } \\
\text { of drugs }\end{array}$} & & & \\
\hline & NNRTI: & NRTI: & PI: \\
\hline & Nevirapine & $\begin{array}{l}\text { Lamivudine/Emtracitabine } \\
\text { Abacavir/Tenofovir }\end{array}$ & 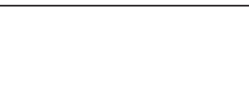 \\
\hline & Efavirenz & Zidovudine & $\begin{array}{l}\text { Atazanavir (boosted) } \\
\text { Saquinavir (boosted) }\end{array}$ \\
\hline & & Didanosine & $\begin{array}{l}\text { Lopinavir (boosted) } \\
\text { Darunavir (boosted) }\end{array}$ \\
\hline & & Stavudine & Indinavir (boosted) \\
\hline & & & Tipranavir (boosted) \\
\hline & & & Ritonavir (boosted) \\
\hline
\end{tabular}

Fig. 1. Metabolic impact of ART classes and individual drugs. ${ }^{[8]}$ NNTRI = non-nucleoside reverse transcriptase inhibitor; NRTI = nucleoside reverse transcriptase inhibitor; $\mathrm{Pl}=$ protease inhibitor.

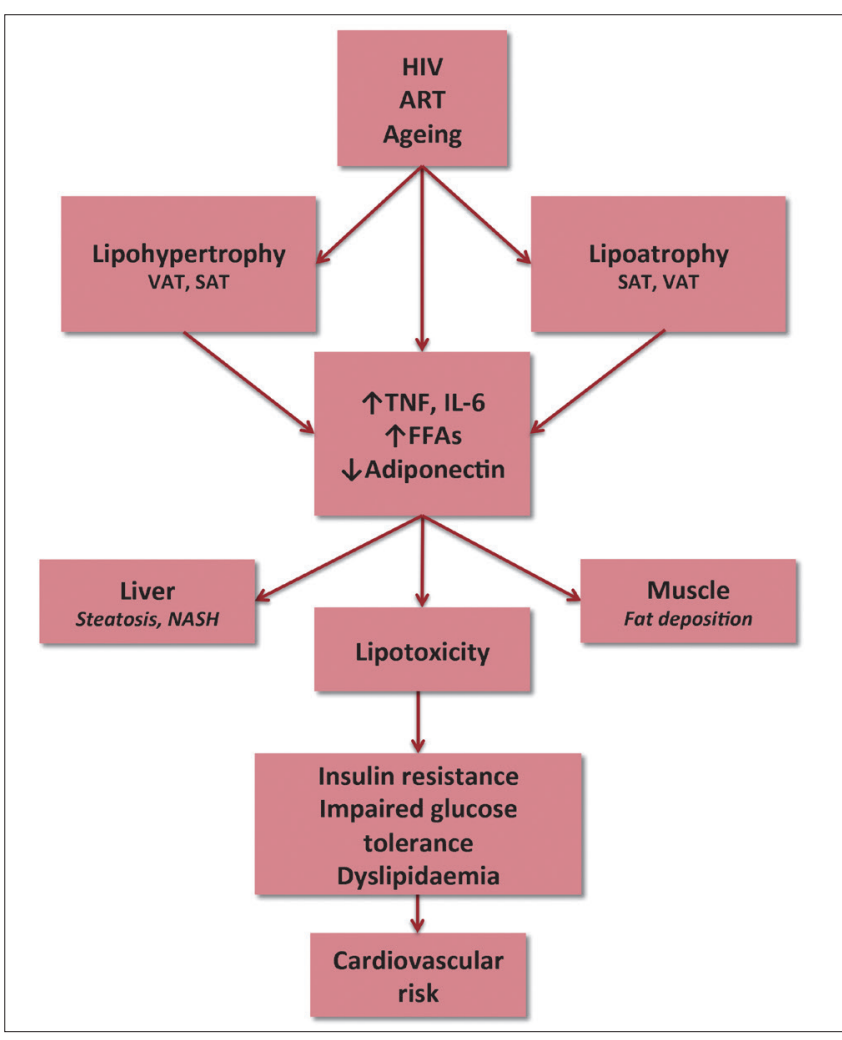

Fig. 2. Metabolic consequences of lipodystrophy. VAT = visceral adipose tissue; $S A T=$ subcutaneous adipose tissue; $T N F=$ tumour necrosis factor; IL-6 = interleukin-6; FFAs = free fatty acids; NASH = Non-alcoholic steatohepatitis.

\section{Management of lipodystrophy Changing ART Lipoatrophy}

The only ART modification that has been shown to partially reverse SAT loss is switching from $\mathrm{d} 4 \mathrm{~T}$ or $\mathrm{AZT}$ to $\mathrm{ABC}$ or $\mathrm{TDF} .^{[11,12]}$ One small study comparing the effect of three $\mathrm{ABC}$ substitution approaches over 48 weeks showed that fat mass in the arms and legs improved significantly $(41 \%$ and $52 \%$, respectively) when $\mathrm{d} 4 \mathrm{~T}$ was replaced with $\mathrm{ABC}$. There was, however, no improvement when treatment with a PI or NNRTI was stopped in favour of $\mathrm{ABC} \cdot{ }^{[13]}$ On average, an estimated $400-500 \mathrm{~g}$ fat can be regained per year, but it is important to bear in mind that some patients may fail to demonstrate significant improvement due to possible exhaustion of the fat mesenchymal stem-cell pool. Furthermore, treatment modification may introduce risks associated with the new $\mathrm{ART}$ regimen, such as $\mathrm{ABC}$ hypersensitivity and TDF-associated nephrotoxicity; therefore, adequate monitoring should be in place. In light of the ongoing controversy regarding $\mathrm{ABC}$ and cardiovascular risk, $\mathrm{ABC}$ may best be avoided in patients with established cardiovascular disease (CVD). An alternative strategy is to switch to an NRTI-sparing regimen. This approach has also been shown to increase total limb fat by approximately $400-500 \mathrm{~g}$ per year, but has the disadvantage of possible increased dyslipidaemia (with all PIs except ATV). Furthermore, data on its long-term virological efficacy is limited. ${ }^{[8,14]}$

\section{Lipohypertrophy}

Weight gain is an expected consequence of successful ART. Weight reduction - or even better, avoidance of weight gain - through healthy diet and exercise may decrease VAT deposition and possibly improve insulin sensitivity and the lipid profile. There is, however, no information about the sufficient amount of diet and exercise needed to maintain this effect, and it may even aggravate LA. There are limited data on the effect of an ART regimen change on LH. In a trial of 201 HIV-infected patients with abdominal fat accumulation and viral suppression, a switch from a twice-daily ritonavir-boosted PI regimen to once-daily ritonavir-boosted ATV led to a non-significant difference in limb fat loss at 48 weeks. ${ }^{[15]}$ Some anecdotal data do, however, hint that ATV may have some effect: a very small case series of three patients described regression of central fat accumulation after nelfinavir (NFV) was replaced with ATV ${ }^{[16]}$ 
In a recent study by Lake et al., ${ }^{[1]]} 39$ virologically controlled women receiving TDF-FTC/ABC-3TC and either NNRTI- or PI-based ART, with $\mathrm{LH}$, were randomised to immediate or delayed switch of the NNRTI or PI component to RAL. After 24 weeks, no statistically significant changes in VAT or SAT, anthropometrics, body mass index (BMI), glucose or C-reactive protein (CRP) were observed, but there were significant improvements in total and low-density lipoprotein (LDL) cholesterol $(p=0.04) \cdot{ }^{\left[{ }^{[7]}\right.}$ There is therefore insufficient evidence to suggest that a change to the newer classes of drugs, such as integrase and entry inhibitors, may reverse LA or LH. The complexity of the data highlights the controversy of changing a treatment regimen for clinical and aesthetic reasons in the face of maximal virological suppression.

\section{Novel treatment strategies Lipoatrophy}

Trials of the thiazolidinediones have shown that this drug class has very modest, if any, effect on lipoatrophic SAT. Although these drugs improve insulin sensitivity, they are known to induce harmful effects on blood lipids. ${ }^{[18]}$ In a randomised controlled trial, pioglitazone was shown to increase the number and function of mitochondria and partially reverse peripheral fat loss in patients without thymidine NRTIs. This effect was, however, not noticeable to the patients. ${ }^{[19]}$ Uridine, a pyrimidine nucleoside, has been postulated to protect fat cells from the adverse effects of thymidine analogues. After initial promising results, however, the drug failed to demonstrate improvement in limb fat in a subsequent multicentre clinical trial of 165 participants. ${ }^{[20]}$ Pravastatin, a lipidlowering 3-hydroxy-3-methyl-glutaryl-CoA (HMG-CoA) reductase inhibitor that is thought to have anti-inflammatory properties, was shown to partially reverse lipodystrophy in 33 hypercholesterolaemic men. ${ }^{[2]]}$ However, it failed to show a beneficial effect in a randomised trial of men who had discontinued thymidine NRTIs. ${ }^{[22]}$

\section{Lipohypertrophy}

Growth hormone $(\mathrm{GH})$ has been shown to decrease VAT, but it may worsen subcutaneous LA and insulin resistance. ${ }^{[8]}$ Tesamorelin, a GHreleasing factor analogue, has been used to restore GH levels and has demonstrated a significant reduction in VAT hypertrophy. It has also been shown to improve the levels of TGs, HDL-C, adiponectin and insulinlike growth factor 1 (IGF-1), although a small but statistically significant worsening of glucose profiles was also evident. Based on these data, the US Food and Drug Administration (FDA) approved tesamorelin for the treatment of excess abdominal fat in HIV-infected patients in November 2010. The drug may only be used in patients without active malignancy and its widespread use is complicated by cost, the need for frequent monitoring of IGF-1 and glycosylated haemoglobin ( $\mathrm{HbA1c}$ ), and the lack of safety data beyond one year of use. Therapy should not be continued for longer than six months in the absence of a favourable treatment response, as assessed by a decrease in waist circumference. ${ }^{[23]}$ Metformin is known to decrease VAT, especially in the presence of insulin resistance. It may, however, worsen subcutaneous LA and should not be used in patients with a low BMI. There are, however, not enough data to recommended its use in patients without diabetes mellitus (DM) at this stage. Anabolic steroids have not shown a beneficial response in the presence of normal blood testosterone levels and can also not be recommended at present.

Overall, in light of the high cost of these treatments, limited data showing minimal improvement, an absence of clear long-term benefits and the possibility of new complications of the therapy, none of these drugs can be recommended for the routine treatment of lipodystrophy at this stage.

\section{Role of surgery Lipoatrophy}

Various surgical interventions have been proposed for the management of facial LA. There are, however, limited long-term data on the different approaches, and inadequate comparisons thereof. Polylactic acid (PLA) is a re-absorbable filler that is immunologically inert and causes only limited inflammation. Most patients report a good response after three to four injections. It is, however, prohibitively expensive. Hyaluronic acid and collagen produce equally favourable results, but the effects are less durable. Transplanting autologous harvested fat cells is becoming increasingly topical, but costs, the invasiveness of the technique and the requirement of general anaesthesia and hospitalisation limit its use. ${ }^{[2]}$ Polyalkylamide (Bio-Alcamid) is a permanent filler and has been used with good effect, especially in cases of severe LA. Permanent fillers do not, however, have long-term safety data and have the disadvantage that, if LA progresses, then the edges of the filler may become visible. Conversely, if fat mass increases, the permanent filler may over-correct the original defect and hence become obvious and unsightly. ${ }^{[24]}$

\section{Lipohypertrophy}

Surgical interventions have been used for localised forms of $\mathrm{LH}$, such as lipomas and buffalo humps. Options include standard surgical removal and liposuction. The duration of effect is, however, variable and up to half of the patients with dorsocervical disease experience a recurrence after $1-2$ years. Surgery can also be considered when significant fat has accumulated around the breast tissue. Breast reduction surgery is invasive, requires anaesthesia and hospitilisation, and has a similar risk of fat return, especially if the patient cannot be established on a PI-sparing regimen. Surgery is not an option for patients with abdominal LH.

\section{Screening for and managing metabolic complications Suggested approach}

Prevention of HIV-related lipodystrophy is the best strategy and all HIVinfected persons should be screened at regular intervals for a history of metabolic disease, dyslipidaemia, DM, hypertension and alteration of body composition (Table 2). Interventions to prevent CVD should vary in intensity according to a patient's absolute risk of ischaemic heart disease. A comprehensive, multi-disciplinary approach is preferred. This should start with lifestyle interventions - counselling to stop smoking, modified diet and regular exercise - and be followed with a change of ART if needed, and the use of lipid-lowering medication in high-risk patients. The prevention and management of type $2 \mathrm{DM}$ and hypertension should be in accordance with guidelines used in the general population. When pharmacological interventions are considered, care should be taken to avoid detrimental pharmacokinetic interactions, such as between statins and PIs. ${ }^{[24]}$

\section{Conclusion}

Lipodystrophy remains a challenge in the long-term management of HIV-infected patients receiving ART, and should be regarded as part of a more pervasive pathology. We recommend approaching the condition with targeted annual screening. In light of the absence of 


\section{Table 2. Suggested annual screening for metabolic complications}

\begin{tabular}{|c|c|}
\hline History & $\begin{array}{l}\text { - Family or personal history of CVD, } \\
\text { DM or HT } \\
\text { - Concomitant treatment for DM, HT } \\
\text { or dyslipidaemia } \\
\text { - Concomitant use of medication with } \\
\text { risk for DM or dyslipidaemia } \\
\text { - Lifestyle: smoking, alcohol, exercise, } \\
\text { diet } \\
\text { - Patient perception of change in body } \\
\text { composition }\end{array}$ \\
\hline Examination & $\begin{array}{l}\text { - Body composition } \\
\text { - BMI } \\
\text { - Waist circumference } \\
\text { - Waist:hip ratio } \\
\text { - Clinical signs of lipodystrophy } \\
\text { - Blood pressure } \\
\text { - Cardiovascular risk assessment }\end{array}$ \\
\hline Bloods & $\begin{array}{l}\text { - Lipids: fasting total cholesterol, TGs, } \\
\text { LDL-C and HDL-C } \\
\text { - Glucose (fasting) } \\
\text { - Liver enzymes: ALT, AST, GGT, ALP } \\
\text { - Renal function: eGFR }\end{array}$ \\
\hline Special investigations & $\begin{array}{l}\text { - ECG: men aged }>40 \text { years and women } \\
\text { aged }>50 \text { years }\end{array}$ \\
\hline \multicolumn{2}{|c|}{ 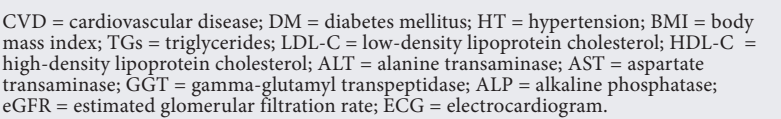 } \\
\hline
\end{tabular}

cost-effective measures to treat LA and $\mathrm{LH}$, prevention remains the best option. Healthcare workers should be sensitised to the early detection of lipodystrophy in patients on thymidine-based ART regimens. Furthermore, affected patients should be switched to an appropriate regimen as soon as is feasible. There is currently no evidence to support the use of new-generation ARVs, except in patients with significant hypercholesterolaemia, where ATV and RAL may present better options.

\section{References}

1. Grinspoon S, Carr A. Cardiovascular risk and body-fat abnormalities in HIVinfected adults. N Engl J Med 2005;352:48-62. [http://dx.doi.org/10.1056/ NEJMra041811]

2. Caron-Debarle M, Lagathu C, Boccara F, Vigouroux C, Capeau J. Feature Review - HIV-associated lipodystrophy: From fat injury to premature aging. Trends Mol Med 2010;16(5):218-229. [http://dx.doi.org/10.1016/j.molmed.2010.03.002]

3. Villarroya F, Domingo P, Giralt M. Drug-induced lipotoxicity: Lipodystrophy associated with HIV-1 infection and antiretroviral treatment. Biochem Biophys Acta 2010;1801(3):392-399. [http://dx.doi.org/10.1016/j.bbalip.2009.09.018]

4. Panel on Antiretroviral Guidelines for Adults and Adolescents. Guidelines for the use of antiretroviral agents in HIV-1-infected adults and adolescents. Bethesda, Maryland, USA: Department of Health and Human Services, 2011:1-166. http://www.aidsinfo.nih. gov/ContentFiles/AdultandAdolescentGL.pdf (accessed 12 June 2012).
5. Nguyen A, Calmy A, Schiffer V, et al. Lipodystrophy and weight changes: Data from the Swiss HIV Cohort Study, 2000 - 2006. HIV Med 2008;9(3):142-150. [http:// onlinelibrary.wiley.com/doi/10.1111/j.1468-1293.2007.00537.x/abstract]

6. Haubrich RH, Riddler SA, DiRienzo G, et al. Metabolic outcomes in a randomized trial of nucleoside, nonnucleoside and protease inhibitor-sparing Regimens for initial HIV treatment. AIDS 2009;23(9):1109-1118. [http://dx.doi.org/10.1097/ QAD.0b013e32832b4377]

7. Jemsek JG, Arathoon E, Arlotti M, et al. Body fat and other metabolic effects of atazanavir and efavirenz, each administered in combination with zidovudine plus lamivudine, in antiretroviral-naive HIV-infected patients. Clin Infect Dis 2006;42(2):273-280. [http://dx.doi.org/10.1086/498505]

8. Lundgren JD, Battegay M, Behrens G, et al. European AIDS Clinical Society (EACS) guidelines on the prevention and management of metabolic diseases in HIV. HIV Med 2008;9(2):72-81. [http://dx.doi.org/10.1111/ j.1468 1293.2007.00534.x]

9. Power R, Tate HL, McGill SM, Taylor C. A qualitative study of the psychosocial implications of lipodystrophy syndrome on HIV positive individuals. Sex Transm Infect 2003;79:137-141. [http://dx.doi.org/10.1136/sti.79.2.137]

10. Martínez E, Garcia-Viejo MA, Blanch J, Gatell JM. Lipodystrophy syndrome in patients with HIV infection: Quality of life issues. Drug Saf 2001;24(3):157-166.

11. Drechsler H, Powderly WG. Switching effective antiretroviral therapy: A review. Clin Infect Dis 2002;35(10):1219-1230. [http://dx.doi.org/10.1086/343050]

12. Moyle GI, Sabin CA, Cartledge J, et al. A randomized comparative trial of tenofovir DF or abacavir as replacement for a thymidine analogue in persons with lipoatrophy. AIDS 2006;20(16):2043.

13. Moyle GI, Baldwin C, Langroudi B, Mandalia S, Gazzard BG. A 48-Week, randomized, open-label comparison of three abacavir-based substitution approaches in the management of dyslipidemia and peripheral lipoatrophy. Acquir Immune Defic Syndr 2003;33(1):22-28.

14. Tebas $\mathrm{P}$, Zhang J, Yarasheski K, et al. Switching to a protease inhibitor-containing, nucleoside-sparing regimen (lopinavir/ritonavir plus efavirenz) increases limb fat but raises serum lipid levels: Results of a prospective randomized trial (AIDS clinical trial group 5125s). J Acquir Immune Defic Syndr 2007;45(2):193. [http:// dx.doi.org/10.1097/QAI.0b013e318042e204]

15. Moyle GJ, Andrade-Villanueva J, Girard PM, et al. A randomized comparative 96-week trial of boosted atazanavir versus continued boosted protease inhibitor in HIV-1 patients with abdominal adiposity. Antivir Ther 2012;17(4):689. [http:// dx.doi.org/10.3851/IMP2083]

16. Haerter G, Manfras BJ, Mueller M, Kern P, Trein A. Regression of lipodystrophy in HIV-infected patients under therapy with the new protease inhibitor atazanavir. AIDS 2004;18(6):952-998.

17. Lake JE, McComsey GA, Hulgan TM, et al. A randomized trial of raltegravir replacement for protease inhibitor or non-nucleoside reverse transcriptase inhibitor in HIV-infected women with lipohypertrophy. AIDS Patient Care STDs 2012,26(9):532-540. [http://dx.doi.org/10.1089/apc.2012.0135]

18. Sutinen J. The effects of thiazolidinediones on metabolic complications and lipodystrophy in HIV-infected patients (Review). PPAR Research 2009:373524. [http://dx.doi.org/10.1155/2009/373524]

19. Slama L, Lanoy E, Valantin M-A, et al. Effect of pioglitazone on HIV-1-related lipodystrophy: A randomized double-blind placebo-controlled trial (ANRS 113). Antivir Ther 2008;13(1):67-76.

20. McComsey G, O'Riordan MA, Choi J, et al. A 48-week randomized study of uridine supplementation vs. switch to TDF on limb fat, mitochondrial function, inflammation and bone mineral density in HIV lipoatrophy. 17th Conference on Retroviruses and Opportunistic Infections, San Francisco, CA, USA, 2010;342.

21. Mallon PWG, Miller J, Kovacic JC, et al. Effect of pravastatin on body composition and markers of cardiovascular disease in HIV-infected men - A randomized, placebo-controlled study. AIDS 2006;20(7):1003-1010. [http://dx.doi. org/10.1097/01.aids.0000222072.37749.5a]

22. Calmy A, Bloch M, Wand $\mathrm{H}$, et al. No significant effect of uridine or pravastatin treatment for HIV lipoatrophy in men who have ceased thymidine analogue nucleoside reverse transcriptase inhibitor therapy: A randomized trial. HIV Med 2010;11(8):493-501. [http://dx.doi.org/10.1111/j.1468-1293.2009.00817.x]

23. Falutz J, Mamputu JC, Potvin D, et al. Effects of tesamorelin (TH9507), a growth hormone-releasing factor analog, in human immunodeficiency virus-infected patients with excess abdominal fat: A pooled analysis of two multicenter, doubleblind placebo-controlled phase 3 trials with safety extension data. J Clin Endocrinol Metab 2010; 95:4291. [http://dx.doi.org/10.1210/jc.2010-0490]

24. Gazzard BG; BHIVA Treatment Guidelines Writing Group. British HIV Association guidelines for the treatment of HIV-1-infected adults with antiretroviral therapy 2008. HIV Med 2008;9(8):563-608. 